\title{
Generative processes in character classification: II. A refined testing procedure
}

\author{
JOHN G. SEAMON \\ Wesleyan University, Middletown, Connecticut 06457
}

\begin{abstract}
Previous work has shown that generative processes have an effect upon reaction time primarily in the time it takes to encode the probe in a character classification task. This experiment extended that finding by examining the performance of two subjects over 12 sessions with precise temporal positioning of the probe character within the generative sequence of the target characters. The results suggest that probe encoding time is influenced by both facilitation and interference effects. The temporal duration of probe pattern recognition is reduced when the probe and the last generated representation are the same, and lengthened when they are different. These effects are lost over a 1-sec interval.
\end{abstract}

A recent examination of the role of rehearsal in a varied-set character classification task found evidence to suggest that rehearsal may reduce the duration of the probe encoding stage through some mechanism of pathway activation by providing the subject with a generated representation of what may appear next as a probe (Seamon \& Wright, 1976). The present experiment sought to improve on the earlier testing format by externally pacing the rehearsal of the target characters by a recurring visual dot. The use of an external cue permitted an evaluation of how accurately people could monitor their rehearsal and, most importantly, it enabled probes to be presented very precisely in relation to the rehearsed characters. If subjects can rehearse targets in time with the external cue, it should be possible to arrange conditions such that the frequency and location of trials in which a person is set for a particular probe can be determined in advance rather than left to chance. Moreover, probing at different points in time during the rehearsal of the target characters may help specify the temporal duration of the previously observed effect of set on probe encoding.

\section{METHOD}

\section{Subjects}

The subjects were two Wesleyan undergraduates who served as paid volunteers and the experimenter. All of the subjects performed in at least several sessions in classification tasks employing words and letters and may, therefore, be considered to be highly familiar with the general classification paradigm.

\section{Apparatus}

The apparatus, materials, and experimental environment were the same as those used previously (Seamon \& Wright, 1976).

This research was supported by NIMH Grant 1-RO3MH27126-01 awarded to the author. Appreciation is expressed to David Todd and Alvin Lucier for making the facilities at the University Computer Center available. Requests for reprints should be sent to John G. Seamon, Department of Psychology, Wesleyan University, Middletown, Connecticut 06457.

\section{Procedure}

The experiment was conducted over 13 sessions, with the first session consisting of 100 trials considered practice and not included in the data analysis. The remaining 12 sessions of approximately 120 trials each were paired so that subjects participated in two sessions per day for 2 days per week. A $1 / 2$-h rest period separated each session on a given day.

Over the 12 experimental sessions, each subject was presented with 1,404 trials consisting of 468 trials for each target set size of 1 , two, and three characters, with half of the correct responses in each target set size positive and half negative. Trials in which subjects made errors were repeated at a randomly determined point. For positive responses, all serial positions within the target array were equally probable.

A trial consisted of an auditory beep followed $.5 \mathrm{sec}$ later by the sequential presentation of the one, two, or three characters in the target array for $1 \mathrm{sec}$ each at a point in the top center of a GT-40 display screen. The last character was followed by a visual mask for 1 sec which blanked out the character space just employed. This served to stop the display of the last character and inform the subject that the preceding characters would form the target array for the current trial. Depending upon target size, a sequential series of from one to five (Target Size 1), one to nine (Target Size 2), or one to thirteen (Target Size 3) recurring dots then appeared on the screen in the position just occupied by the mask. Each dot was shown for $1 \mathrm{sec}$, with its displayed intensity decremented in a stepwise fashion from bright to very dim over each 1-sec interval. The recurring dots served as a rehearsal cue for the subject, who was required to rehearse subvocally by mouthing the target characters cyclically in the order shown at a rate of $1 \mathrm{sec}$ per character and in pace with the dots, e.g., the first target character was rehearsed with the presentation of the first dot, etc. Somewhere during the rehearsal sequence, but never before each target character had been rehearsed at least twice, a probe character replaced a dot in the same location on the display screen. The intensity of the probe character did not vary and was the same as that used for the target set and the brightest period of a dot exposure. For a given dot duration of $1 \mathrm{sec}$, a letter probe could replace a dot at either its onset time or halfway through the 1-sec interval. Probe position in the rehearsal sequence was equally probable both between dots in the sequence and within the dot intervals and was, like target set size, response, and serial position in a target for positive probes, randomized from trial to trial.

After making a manual response to the probe, the subject was presented with a listing in multiple-choice format of all of the target characters from the array just shown, along with an additional choice labeled "DON'T KNOW." The subject's task was to select the number of the alternative that corresponded to the item in the target array which was the last item rehearsed prior to the 


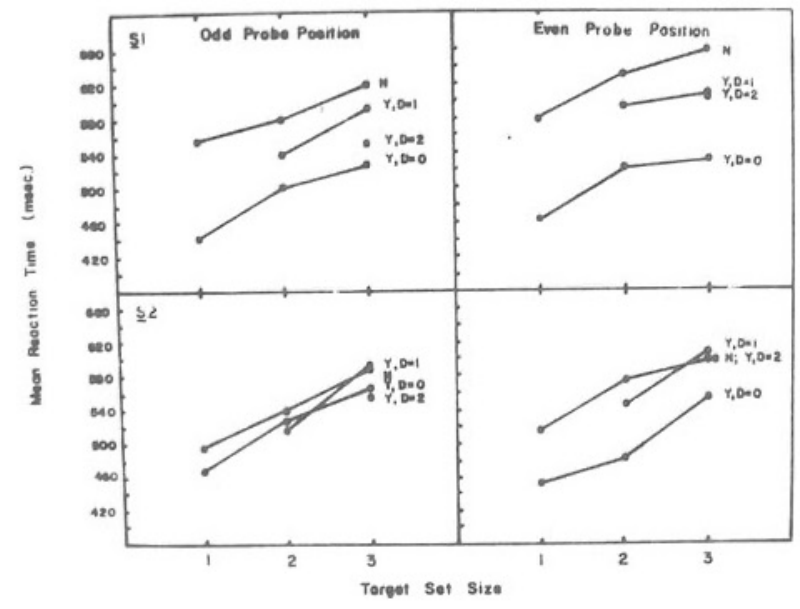

Figure 1. Mean RT as a function of target set size, response, rehearsal distance function, probe position, and subject.

presentation of the probe. If the subject was unsure of his last rehearsal or otherwise unable to make an appropriate character selection. he was instructed to select the DON'T KNOW alternative. The responses to the multiple-choice test were made manually via the subject's keyboard, with a visual prompt presented on the screen if the selection had not been made within $5 \mathrm{sec}$. The rehearsal question was followed by a 2 -sec intertrial interval and the next trial.

\section{RESULTS AND DISCUSSION}

All analyses were performed on an individualsubject basis, with the data from one of the subjects excluded due to the discovery that he failed to use the last rehearsal identification rule consistently and correctly.

The percentage of last rehearsal choices as a function of target set size, item, and probe position is shown in Table 1. The rehearsal patterns for each subject are strikingly similar across all target set sizes. With only one item to rehearse in Target Size 1, the subjects invariably selected that item as their last rehearsal over all tive possible probe positions. However, with Target Sizes 2 and 3, a definite rehearsal pattern emerges. Table 1 shows that the subjects anticipated the dot cue by rehearsing the next item in the rehearsal sequence before its corresponding dot had appeared. This is seen by a shift in the last rehearsal selection to the next item in the target on the even-numbered probe positions. These rehearsal patterns, based on the most frequently selected last rehearsal choices, indicate that, while there is a high degree of correspondence at dot onset (odd-numbered probe positions) between where a subject should be in the rehearsal sequence and where a subject actually is, probe position per se cannot completely specify the last rehearsal. A last rehearsal identification is necessary for those relatively few instances when subjects either change their rehearsal pattern or start rehearsing out of sequence with the dots. Not shown in Table 1 are the percentage of DON'T KNOW selections which were extremely rare. Subject 1 chose this alternative in $.22 \%$ of the trials, while Subject 2 never chose it.

Mean latencies for correct responses for each target set size, response condition, rehearsal distance function, and odd or even probe position are presented in Figure 1. Mean RTs for odd- or even-numbered probe positions were examined separately to determine if probing at dot onset (odd probe positions) or dimunition (even probe positions) had an effect upon performance. The rehearsal distance function for positive responses was defined (Seamon \& Wright, 1976) as the computed circular distance between the probe character and the last character rehearsed, such that the distance (D) is 0 when these characters are the same, 1 when the probe is the character rehearsed just previous to the last rehearsal item, and so on.

The data from Subject 1 indicate that RTs were

Table I

Percentage of Last Rehearsals for Each Target Item by Probe Position and Target Set Size

\begin{tabular}{|c|c|c|c|c|c|c|c|c|c|c|c|c|c|c|}
\hline \multirow{2}{*}{\multicolumn{2}{|c|}{$\begin{array}{l}\text { Target Last } \\
\text { Size Rehearsal }\end{array}$}} & \multicolumn{13}{|c|}{ Probe Position } \\
\hline & & 1 & 2 & 3 & 4 & 5 & 6 & 7 & 8 & 9 & 10 & 11 & 12 & 13 \\
\hline \multicolumn{15}{|c|}{ Subject 1} \\
\hline 1 & Item 1 & 100 & 100 & 100 & 98.9 & 100 & & & & & & & & \\
\hline \multirow[t]{2}{*}{2} & Item 1 & 85.7 & 4.1 & 26.9 & 98.1 & 84.0 & 0 & 23.1 & 96.2 & 76.1 & & & & \\
\hline & Item 2 & 14.3 & 95.9 & 71.2 & 1.9 & 14.0 & 100 & 76.9 & 3.8 & 23.9 & & & & \\
\hline \multirow[t]{3}{*}{3} & Item 1 & 91.7 & 2.8 & 0 & 2.8 & 11.1 & 97.1 & 75.0 & 2.9 & 0 & 0 & 8.3 & 90.9 & 91.4 \\
\hline & Item 2 & 8.3 & 97.2 & 85.7 & 8.3 & 0 & 0 & 25.0 & 97.1 & 100 & 0 & 2.8 & 0 & 8.6 \\
\hline & Item 3 & 0 & 0 & 14.3 & 88.9 & 88.9 & 2.9 & 0 & 0 & 0 & 100 & 88.9 & 9.1 & 0 \\
\hline \multicolumn{15}{|c|}{ Subject 2} \\
\hline 1 & Item I & 100 & 100 & 100 & 100 & 100 & & & & & & & & \\
\hline \multirow[t]{2}{*}{2} & Item 1 & 100 & 0 & 7.8 & 100 & 98.1 & 2.0 & 4.2 & 98.0 & 100 & & & & \\
\hline & Item 2 & 0 & 100 & 92.2 & 0 & 1.9 & 98.0 & 95.8 & 2.0 & 0 & & & & \\
\hline \multirow[t]{3}{*}{3} & Item 1 & 100 & 0 & 0 & 0 & 2.8 & 100 & 100 & 0 & 0 & 0 & 0 & 100 & 97.0 \\
\hline & Item 2 & 0 & 100 & 100 & 0 & 0 & 0 & 0 & 100 & 100 & 2.8 & 0 & 0 & 3.0 \\
\hline & Item 3 & 0 & 0 & 0 & 100 & 97.2 & 0 & 0 & 0 & 0 & 97.2 & 100 & 0 & 0 \\
\hline
\end{tabular}


generally faster at odd- than at even-numbered probe positions and that RTs for positive responses were influenced by the rehearsal distance function, with fastest RTs when $D=0$ at all probe positions and target set sizes. The nine means shown for Subject 1 for the odd probe positions are lower than their corresponding means for the even probe positions in each instance, with the overall mean RTs of 563 and $598 \mathrm{msec}$ ( $\mathrm{SEs}=3$ and $4 \mathrm{msec}$ ) for odd and even probe positions, respectively, strongly indicating that Subject 1 was faster to probes at dot onset than dot dimunition. Confidence intervals were calculated for each mean when $D=0,1$, or 2 for both types of probe positions. For the odd probe positions there is no overlap in the .95 confidence intervals for $D=0$ and $\mathrm{D}=1$ at Set Size 3, while some overlap in intervals is present for $D=0$ and $D=2$ at Set Size 3 and $D=0$ and $D=1$ at Set Size 2. The latter difference between the $\mathrm{D}=0$ and $\mathrm{D}=1$ means is, however, significant, $t=2.50, \mathrm{p}<.01$. For the even probe positions there is no overlap in the .95 confidence intervals for $D=0$ and $D=1$ at Set Size $2, D=0$ and $D=1$ at Set Size 3 , and $D=0$ and $D=2$ at Set Size 3. Taken together, the results of Subject 1 indicate a specific effect of set or generative processes, such that RT is fastest when the probe character is the same as the last character rehearsed, and a general effect of alterness by this subject being faster to all probes at dot onset than diminution. The differentiation of alterness from set is consistent with Posner and Boies (1971) and suggests that a person may be simultaneously alert for any stimulus and set for a particular stimulus.

Interestingly, RT serial position effects like those observed previously (Seamon \& Wright, 1976) were obtained for the first subject and, to a lesser extent, for the second subject. No adequate explanation is available, although such effects in the present experiment may not be attributed to either differential distributions of $D$ valves between serial positions or a speed-accuracy tradeoff (Pachella, 1974; Wickelgren, Note 1).

Important differences between subjects may be seen in Figure 1. For Subject 2, there is a selective, rather than an overall, increase in RT from odd to even probe positions and an effect of set that is present only on even probe positions. Mean RTs interacted with probe position such that RTs for negative responses or positive responses when $D=1$ or 2 increased from odd to even probe positions in all instances, while RTs for positive responses when $\mathrm{D}=0$ decreased in each case. The overall mean RTs for negative responses and positive responses when $\mathrm{D}=1$ or 2 were 565 and $592 \mathrm{msec}$ ( $\mathrm{SEs}=5$ and $6 \mathrm{msec}$ ) for the odd and even probe positions, respectively, while for $\mathrm{D}=0$ the corresponding means were 525 and $502 \mathrm{msec}$ (SEs = 7 and $8 \mathrm{msec}$ ). Calculation of the .95 confidence intervals around each positive response mean for the odd probe positions show considerable overlap in the intervals for the $D=0$ and $D=1$ or 2 means at Set Sizes 2 and 3 . For the even probe positions there is no overlap in the intervals for the $D=0$ and $D=1$ means at Set Size 2, but some overlap in the comparisons of D $=0$ and D $=1$ or 2 at Set Size 3 . Both of these latter comparisons, however, yielded a significant difference between the means, ps $<.05$.

Unlike the results of Subject 1, the data from Subject 2 do not show a general alerting function for the dot onset periods. Rather, a dot onset period seemed to be a neutral state for this subject in which he was apparently not set for anything. At even probe positions, however, the effect of set is once more apparent as RTs are reliably faster for $\mathrm{D}=0$ means than all other conditions. The presence of a neutral state is important, as it permits a determination of the role of generative processes in this task. Comparing the neutral state to the set state for $D=0$ shows a reduction in $\mathrm{RTs}$ indicative of a facilitation effect, while a corresponding interference effect is evidenced by an increase in RT for all other conditions.

An examination of the error percentages for each of the mean RTs in Figure 1 indicates that the RT differences between the odd and even probe positions and the $D=0$ and $D=1$ or 2 distance functions cannot be attributed to a speed-accuracy trade off. Errors were lower for the $D=0$ than the $D=1$ or 2 conditions. As noted by Wickelgren (Note 1), it is safe to conclude that conditions which yield shorter RTs and lower error rates (e.g., $\mathrm{D}=0$ ) are less difficult than conditions with longer RTs and higher error rates (e.g., $D=1$ or 2 ). Further, the effect of set cannot be attributed to either a response bias (see Seamon \& Wright, 1976) or a difference in search processes (see Klatzky \& Smith, 1972; Miller \& Pachella, 1973; Seamon \& Wright, 1976; Shiffrin \& Schneider, 1974), as the RT functions for D $=0$ are generally parallel to those of the negative responses. Rather, the data suggest that set can either facilitate or interfere with the pattern recognition processes of probe encoding, such that generating the appropriate model beforehand reduces the time it takes to encode the probe character off the display screen, while generating an inappropriate model lengthens that time. Moreover, the finding that RT is generally unaffected by the number of character spaces in the rehearsal sequence between the probe and the last rehearsal when both are different (see also Seamon \& Wright, 1976) suggests that the effect of a generated representation is lost rather quickly: in this case within $1 \mathrm{sec}$. This loss in set effectiveness could reflect 
the potentially interfering activity of generating the next representation or a decay factor which operates over a 1 -sec interval.

\section{REFERENCE NOTE}

1. Wickelgren. W. A. Speed-accuracy tradeoff and information processing dyanmics. Paper presented at the meeting of the Psychonomic Society, Boston, Massachusetts, November 1974.

\section{REFERENCES}

Klatzky, R. L.. \& SMith, E. E. Stimulus expectancy and retrieval from short-term memory. Journal of Experimental Psychology, 1972, 94, 101-107.
Miller, J. O., \& Pachella, R. G. Locus of the stimulus probability effect. Journal of Experimental Psychology, 1973, 101, 227-231.

Pachella, R. G. The interpretation of reaction time in information-processing research. In B. H. Kantowitz (Ed.), Human information processing: Tutorials in performance and cognition. Hillsdale. N. J: Erlbaum. 1974. Pp. 41-82.

Posner, M. I., \& Boles, S. J. Components of attention. Psychological Review, 1971, 78, 391-408.

SeAmon. J. G.. \& Wright, C. E. Generative processes in character classitication: Evidence for a probe encoding set. Memory \& Cognition, 1976, 4, 96-102.

Shiffrin, R. M.. \& SChNeIDER, W. An expectancy model for memory search. Memory \& Cognition. 1974. 2. 616-628.

(Received for publication December 5, 1975.) 